\title{
The new detectors of the KLOE-2 experiment
}

\section{Danilo Domenici* on behalf of the KLOE-2 collaboration ${ }^{\dagger}$}

Laboratori Nazionali di Frascati, INFN

E-mail: Danile.Domenicidenf.infn.it

\begin{abstract}
Three new sub-detectors have been installed in the KLOE apparatus of the Frascati National Laboratory of INFN, for a new data taking period started in autumn 2013. Photon detection is upgraded by means of a small crystal calorimeter in the very forward direction and of a tungstenplastic scintillator sampling device covering the low-beta quadrupoles of the accelerator. A new tracking device, the first cylindrical GEM ever built, is inserted to extended tracking capabilities closer to the interaction point. The novel idea of a cylindrical GEM was developed at the Frascati Laboratory in order to build a transparent device with a total material budget of only $2 \%$ of a radiation length, thus minimizing the multiple scattering of low-momentum particles. It is composed by four tracking layers with diameters from $260 \mathrm{~mm}$ to $410 \mathrm{~mm}$ and an active length of $700 \mathrm{~mm}$.
\end{abstract}

The European Physical Society Conference on High Energy Physics -EPS-HEP2013

18-24 July 2013

Stockholm, Sweden

${ }^{*}$ Speaker.

${ }^{\dagger}$ D. Babusci, D. Badoni, I. Balwierz-Pytko, G. Bencivenni, C. Bini, C. Bloise, F. Bossi, P. Branchini, A. Budano, L. Caldeira Balkeståhl, G. Capon, F. Ceradini, P. Ciambrone, F. Curciarello, E. Czerwiński, E. Danè, V. De Leo, E. De Lucia, G. De Robertis, A. De Santis, P. De Simone, A. Di Domenico, C. Di Donato, R. Di Salvo, D. Domenici, O. Erriquez, G. Fanizzi, A. Fantini, G. Felici, S. Fiore, P. Franzini, A. Gajos, P. Gauzzi, G. Giardina, S. Giovannella, E. Graziani, F. Happacher, L. Heijkenskjöld, B. Höistad, L. Iafolla, M. Jacewicz, T. Johansson, K. Kacprzak, A. Kupsc, J. Lee-Franzini, B. Leverington, F. Loddo, S. Loffredo, G. Mandaglio, M. Martemianov, M. Martini, M. Mascolo, R. Messi, S. Miscetti, G. Morello, D. Moricciani, P. Moskal, F. Nguyen, A. Palladino, A. Passeri, V. Patera, I. Prado Longhi, A. Ranieri, C. F. Redmer, P. Santangelo, I. Sarra, M. Schioppa, B. Sciascia, M. Silarski, C. Taccini, L. Tortora, G. Venanzoni, W. Wiślicki, M. Wolke, J. Zdebik 


\section{Introduction}

The DAФNE collider at the Frascati National Laboratory has been recently upgraded with a new interaction scheme implementing crab cavities. A total integrated luminosity of about $10 \mathrm{fb}^{-1}$ is foreseen to be delivered to the KLOE- 2 experiment in the next $2 \div 3$ years of data taking.

The KLOE apparatus is composed by two main sub-detectors: a huge Drift Chamber (DC) operated with a helium based gas mixture, and a lead/scintillating fibers calorimeter (EMC). The whole detector is immersed in an axial $0.52 \mathrm{~T}$ magnetic field produced by a superconductive coil. With this set-up the experiment has collected $2.5 \mathrm{fb}^{-1}$ of integrated luminosity at the $\Phi$ peak in the years 2001-2006, accomplishing an extensive program focused on precision kaon and hadron physics [四].

For the next run KLOE has been upgraded with the installation of three new detectors covering the volume close to the DA $\Phi$ NE interaction region:

- Crystal Calorimeter (CCAL): two calorimeters placed on both sides of the interaction point that increase the acceptance for low angle photons thus improving the reconstruction efficiency for rare decays like $K_{S} \rightarrow \gamma \gamma$.

- Quadrupole Calorimeter (QCAL): two calorimeters covering the new focusing QD0 magnet placed in the acceptance of the drift chamber; they are mandatory in order to reduce the background for the CP violating decay $K_{L} \rightarrow \pi^{0} \pi^{0}$.

- Inner Tracker (IT): a gaseous GEM tracker composed by four cylindrical layers surrounding the interaction point; this improves the space resolution of the $K_{S} \rightarrow \pi^{+} \pi^{-}$vertex by a factor of three.

The improvement of the detection performance in the region close to the interaction point will be a great benefit for the new physics program, mainly focused on short-lived particle decays [[]].

\section{Crystal Calorimeter}

The CCAL increases the acceptance of the central calorimeter from $18^{\circ}$ down to $10^{\circ}$, turning out in an enhancement of the discrimination power for the rare $K_{S} \rightarrow \gamma \gamma$ decay over the background mostly given by the $K_{S} \rightarrow \pi^{0} \pi^{0}$ decay, that can mimic the signal when two photons are lost at a small angle [B].

It is composed by two modules with a truncated cone shape, symmetrically positioned $20 \mathrm{~cm}$ from the interaction point, directly around the beam pipe, in front of the permanent quadrupole magnets. (Fig.W).

One module is realized by four aluminum conical sections each covering $90^{\circ}$ of the azimuthal angle. The active part of the detector is represented by 48 LYSO crystals read-out by square Silicon photomultipliers (SiPM) (Fig.D). 


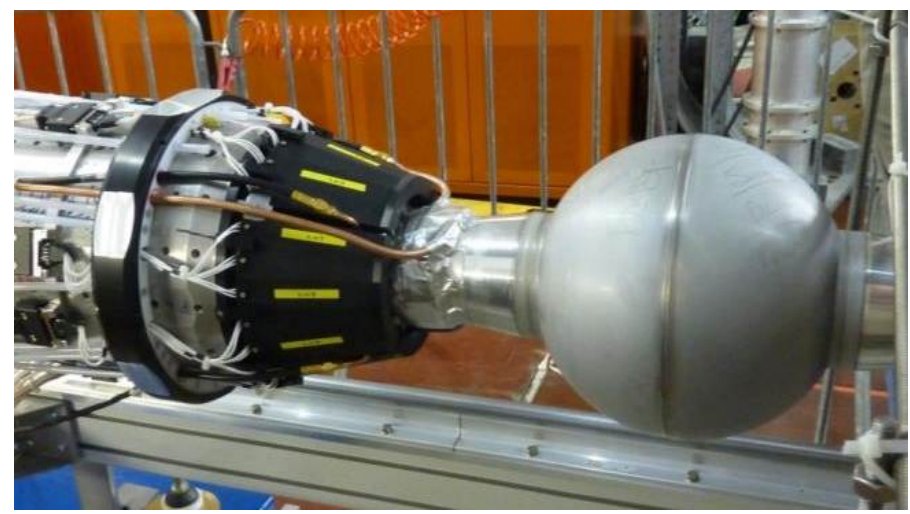

Figure 1: One of the two modules of the CCAL (in black) mounted on the beam-pipe. The Beryllium sphere surrounding the interaction point is visible on the right of the picture.
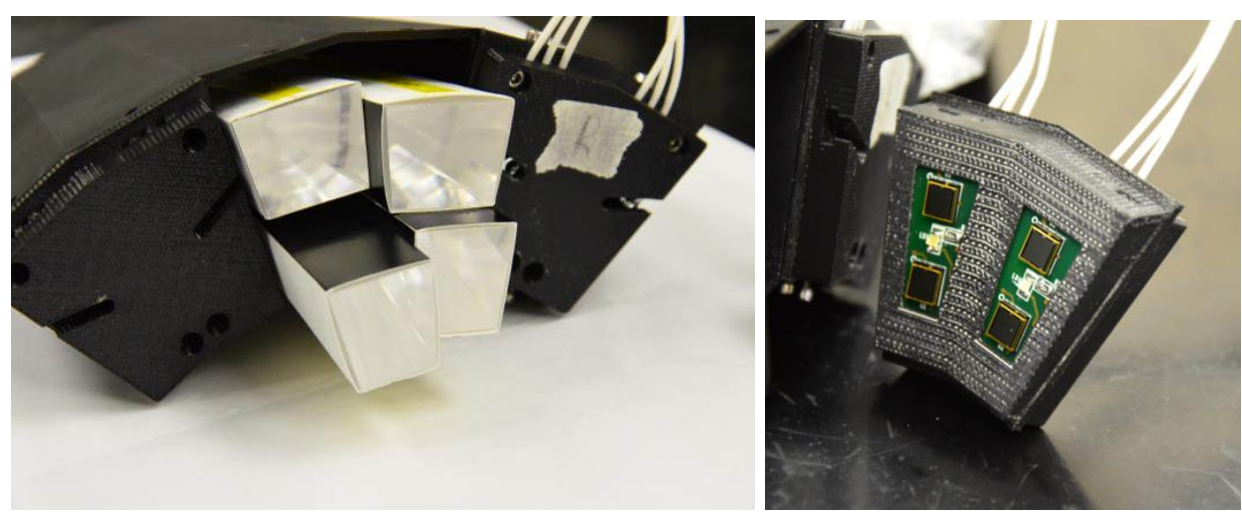

Figure 2: Left: Some of the LYSO crystals inserted in the aluminum structure corresponding to a quarter of a module. Right:the closing plastic case hosting four SiPM and the calibration LEDs.

The main characteristics of the CCAL are:

- A time resolution of $50 \div 120$ ps at energies respectively of $500 \div 100 \mathrm{MeV}$, required for an optimal rejection of the background.

- A space resolution of about $5 \mathrm{~mm}$, given by the crystal size.

- An energy resolution of $15 \%$ at $100 \mathrm{MeV}$, mostly limited by the fact that the shower is not fully contained.

- A thickness corresponding to 7.3 radiation lengths.

- A light yield of 300 photoelectrons per MeV per crystal. 


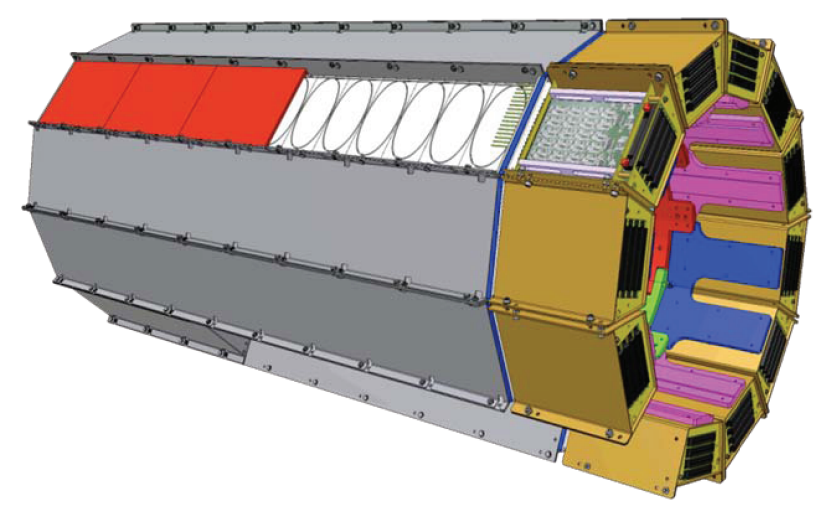

Figure 3: A sketch of the QCAL with an exploded view of one of the 12 modules.

\section{Quadrupole Calorimeter}

The coverage of almost the whole solid angle by the calorimeter system of KLOE is completed by the full instrumentation of the two quadrupole focusing magnets placed at $35 \mathrm{~cm}$ from the interaction point. This hermeticity to the photons is mandatory, for instance, for the study of the CP violating decay $K_{L} \rightarrow \pi^{0} \pi^{0}$, where the most important background source is given by the more abundant $K_{L} \rightarrow \pi^{0} \pi^{0} \pi^{0} \pi^{0}$. Using the QCAL as a veto the background can be rejected while losing only $1 \%$ of the signal [四].

It is composed by two dodecagonal structures surrounding the magnets and the beam-pipe from $35 \mathrm{~cm}$ to the interaction point and $80 \mathrm{~cm}$ long (Fig.[1). Each of the 12 modules is a sampling calorimeter with 16 square towers, with dimensions $5 \times 5 \mathrm{~cm}^{2}$. The sampling structure is realized by 5 layers of scintillating tiles interleaved with 5 layers of Tungsten absorbers. The tiles are coated with reflecting varnish and dug with a circular groove housing wavelength-shifting fibers that route the light toward the back-end of the module. Here the fibers are read-out by circular $(1.2 \mathrm{~mm}$ diameter) SiPM kept in place by a precise positioning Alumina plate coupled to a PCB integrating
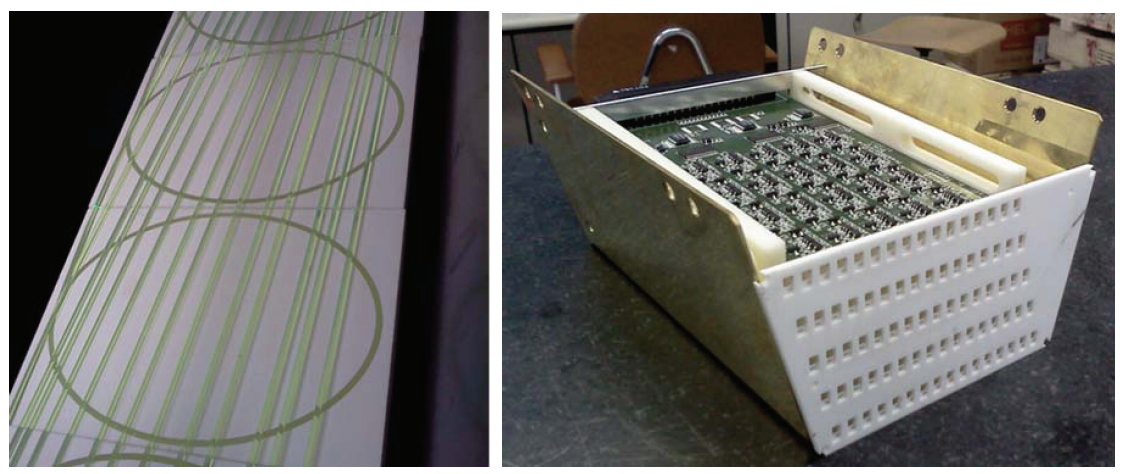

Figure 4: Left: Scintillating tiles painted with reflecting varnish with the WLS fibers. Right: SiPM positioning plate coupled to the FEE board. 
a pre-amplifier and a high-voltage regulator (Fig.(G).

The main characteristics of the QCAL are:

- A time resolution of $750 \mathrm{ps.}$

- A space resolution of $5 \mathrm{~cm} / \sqrt{ } 12$ given by the readout of the $5 \times 5 \mathrm{~cm}^{2}$ tiles.

- A total thickness corresponding to 5 radiation lengths.

- A light yield of 24 photoelectron per MeV per tile.

\section{Inner Tracker}

The IT will enhance the tracking capability of the Drift Chamber, increasing the sampling length as close as $13 \mathrm{~cm}$ from the interaction point. Both the momentum and space resolutions will benefit of the new detector. For instance the $K_{S} \rightarrow \pi^{+} \pi^{-}$decay vertex resolution, of utmost importance for the interferometric measurements, is foreseen to improve to $c \tau_{S} / 3$, a factor of 3 better than present result [5].

It is composed by four cylindrical GEM detectors with diameters of 26,31,36 and $41 \mathrm{~cm}$, and an active length of $70 \mathrm{~cm}$ along the beam axis. The layout of a cylindrical triple-GEM is shown in Fig.5. The manufacturing technique used to realize all the cylindrical electrodes (GEM, cathode

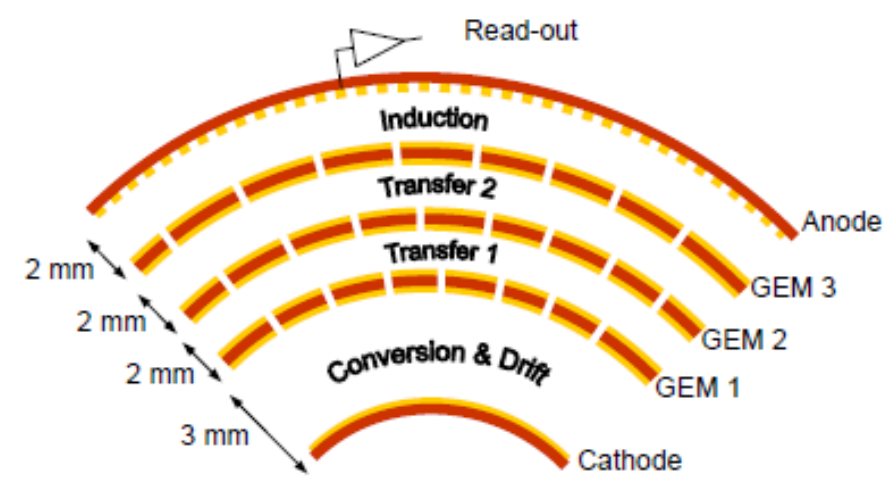

Figure 5: Layout of a cylindrical triple-GEM.

and read-out planes) has been specifically developed at the LNF for the KLOE-2 IT, exploiting some of the unique characteristics of the polyimide based micro-pattern gas detectors, like the intrinsic light-weight structure of a GEM foil and its extreme flexibility. We have then been able to limit the total material of the IT to $2 \%$ of a radiation length in all the active area, avoiding frame overlaps thanks to the fully cylindrical structure [圆 [四].

The GEM are operated with a Ar/Isobuthane : 90/10 gas mixture and a gain of about $10^{4}$. A spatial resolution of $\sigma_{r \phi} \simeq 250 \mu \mathrm{m}$ and $\sigma_{Z} \simeq 400 \mu \mathrm{m}$, respectively for the azimuthal angle and the distance along the beam axis, is achieved from the combination of the spatial information of longitudinal $\mathrm{X}$ strips and $25^{\circ}$ angled $\mathrm{V}$ strips. The read-out charge is coupled to a 64 channels 


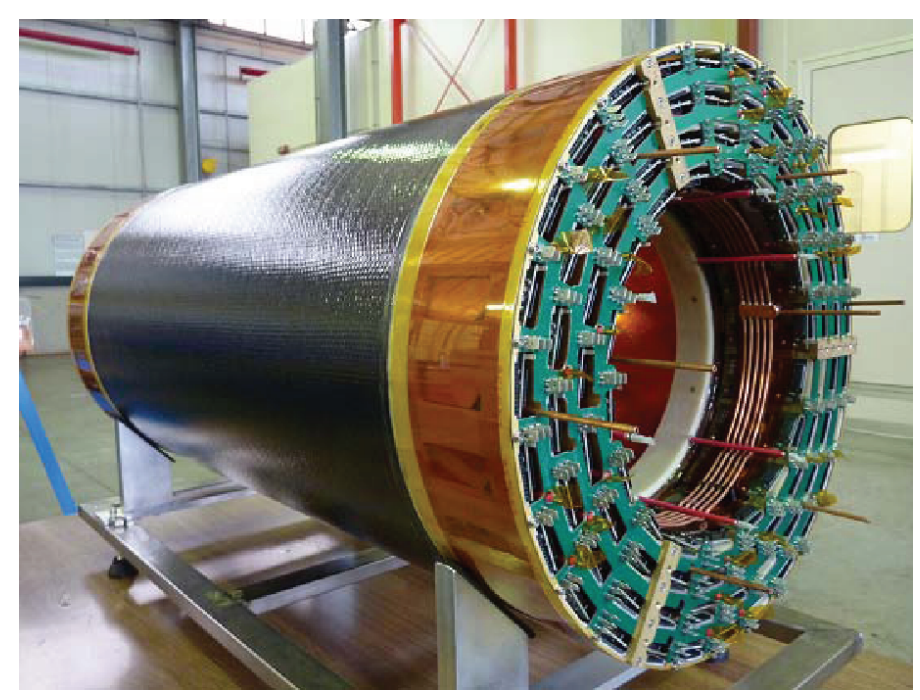

Figure 6: The Inner Tracker.

pre-amplifier with digital output based on the GASTONE chip, an ASIC expressly developed for KLOE-2 [8]. The total number of read-out channels is about 20000.

\section{Integration of the detectors}

The assembling of the DAФNE interaction region started in April, just after the new detectors have been completed. The whole integration procedure, including the insertion inside the KLOE-2 apparatus lasted 3 months. During this period the detectors have been mounted, aligned, cabled and tested with dedicated cosmic rays runs.

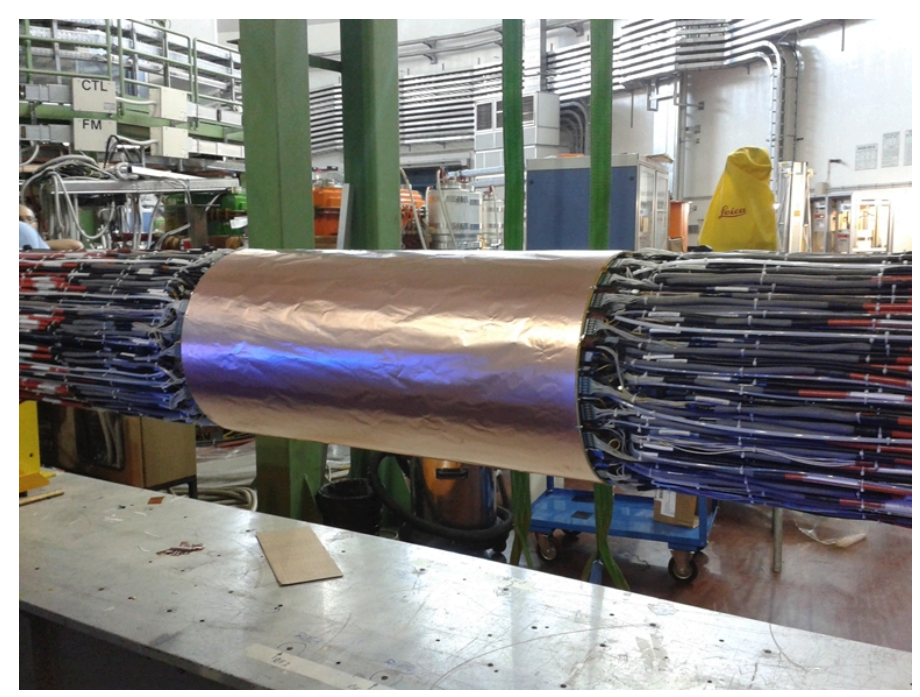

Figure 7: The DAФNE interaction region equipped with the new KLOE-2 detectors. 
Fig. $\square$ shows a part of the beam-pipe equipped with the detectors placed on the assembling bench. In the middle there is the IT wrapped with a $18 \mu \mathrm{m}$ thick Copper shield. Placed aside, the two QCALs are clad by the 400 cables coming out from the IT's ends, providing signals, low voltages, high voltages, gas flushing and air cooling. The CCALs are inside the tracker, close to the interaction point.

\section{Conclusions}

The second run of the KLOE-2 experiment at DAФNE has just started with the aim of 2/3 years of data taking and $10 \mathrm{fb}^{-1}$ of integrated luminosity.

The physics program is vast and includes precision measurements of the rare decays of $K_{S}, \eta$ and $\eta^{\prime}$, detailed study of the basics principles of the quantum mechanics exploiting the interferometry oh the coherent $K^{0} \bar{K}^{0}$ system, and the search for a possible new light boson mediating a hidden force.

Three new detectors have been installed onto the upgraded DAФNE interaction region. The calorimeters will extend the coverage of the main Electromagnetic Calorimeter and the Inner Tracker will work together with the central Drift Chamber in order to improve the reconstruction resolution of vertices close to the interaction point.

The commissioning of the detector is presently underway and the first $e^{+} e^{-}$collisions are foreseen for winter of 2013.

\section{References}

[1] F. Bossi et al., Rivista del Nuovo Cimento, Vol.31, N.10 (2008).

[2] G. Amelino Camelia et al., Eur. Phys. J. C 68 (2010) 619.

[3] F. Happacher et al., Nucl. Phys. Proc. Suppl. 197, (2009) 215.

[4] M. Cordelli et al., Nucl. Instr. and Meth. A 617, (2010) 105.

[5] F. Archilli et al., Technical Design Report of the Inner Tracker for the KLOE-2 experiment, LNF-10/3(P) INFN-LNF, Frascati, 2010.

[6] G. Bencivenni et al., Nucl. Instr. and Meth. A 572 (2007) 168.

[7] G. Bencivenni and D. Domenici, Nucl. Instr. and Meth. A 581 (2007) 221.

[8] A. Balla et al., Nucl. Instr. and Meth. A 604 (2009) 23. 